\title{
José Echegaray, un matemático polifacético: Premio nobel de literatura, político destacadísimo, impulsor de la física y matemática española al final del siglo IXX y de principios del XX
}

José Echegaray, a multi-faceted mathematician: Nobel Prize in Literature, an outstanding politician, promoter of Spanish physics and mathematics at the end of the 20th century and the beginning of the 20th

\author{
Vernor Arguedas \\ vernor.arguedas@ucr.ac.cr \\ Escuela de Matemática \\ Universidad de Costa Rica \\ Costa Rica
}

Resumen. Se discuten algunos aspectos de la riquísima vida de Don José Echegaray, en sus facetas de matemático, político, literario así como culturales en la convulsa España de la segunda mita del S XIX y comienzo del S XX.

Palabras clave: José Echegaray, física matemática, teoría de Galois, trascendencia de pi. Premio Nobel

\begin{abstract}
Some aspects of the rich life of Don José Echegaray are discussed, both mathematical, political, literary as well as cultural in the turbulent Spain of the second half of the 19th century and the beginning of the 20th century.
\end{abstract}

KeyWords: José Echegaray, mathematical physics, Galois Theory, Pi transcendence, Nobel price 


\section{1}

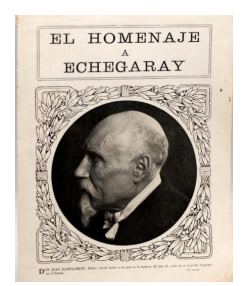

José Echegaray nació en la calle del Niño (hoy calle Quevedo), en Madrid,el 19 de abril de 1832. Hijo de José Echegaray Lacosta, médico aragonés, y de Manuela Eizaguirre Chale, navarra, permaneció en Madrid hasta los cinco años, momento en que se trasladó a Murcia ya que su padre pasó a esa ciudad como profesor de Instituto, para enseñar Botánica y Agricultura. En Murcia estudió el Bachillerato, que completó en junio de 1846,con la calificación de Sobresaliente.

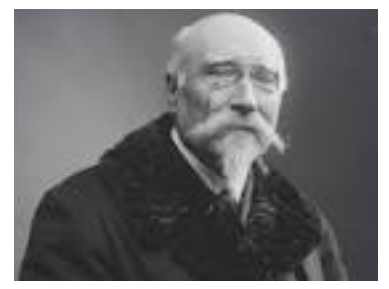

Tras finalizar el Bachillerato en Murcia, y pasar un año preparándose, se trasladó (un viaje de quince días, en una diligencia arrastrada por mulas), en agosto de 1848 a Madrid, a estudiar en la Escuela de Caminos, una elección no demasiado extraña para alguien entre cuyos intereses figuraba la matemática, especialmente si tenemos en cuenta el estado de la enseñanza matemática en la España de mediados del siglo XIX. En efecto, hacia 1850 no existían en España Facultades de Ciencias, estando incluida este tipo de estudios dentro de la Facultad de Filosofía

(fue la ley Moyano de 1857 la que remedió esta situación). El escalafón de catedráticos de la Universidad española de 1847demuestra que el número de catedráticos de matemáticas no era muy elevado. Así, y aunque el propósito de la Escuela de Caminos era formar técnicos (ingenieros) y no matemáticos, la componente matemática existente en ella era lo suficientemente fuerte como para hacer de esa institución uno de los principales centros de enseñanza superior en lo que a las matemáticas se refiere. $\mathrm{Al}$ margen de otras consideraciones, la dimensión matemática en la enseñanza ofrecida en la Escuela de Caminos madrileña es una manifestación del hecho de que en las Escuelas Especiales de Ingeniería españolas de la segunda mitad del siglo XIX la influencia predominante era la de las Escuelas Técnicas francesas, especialmente la Ecole Centrale des Arts et Manufactures y la Ecole Polytechnique, fundadas, respectivamente, en 1829 y 1794. Un ejemplo significativo de la relación existente en España, a mediados del siglo XIX, entre matemáticas e ingenieros de Caminos, es el de la composición de la sección de Ciencias Exactas de la Real Academia de Ciencias Exactas, Físicas y Naturales de Madrid, institución fundada en 1847. De las doce plazas fundacionales de esa sección, seis estaban ocupadas por ingenieros de Caminos, de los cuales cuatro eran o habían sido profesores de la Escuela. José Echegaray, llegaría a a ser un ejemplo más de esta situación.

La Escuela de Caminos, Canales y Puertos en la que estudió Echegaray corresponde a la tercera época. $\mathrm{Al}$ igual que las de ingenieros de Minas, Industriales, Agrícolas y Montes, la apertura de estas Escuelas forma parte de la remodelación institucional que siguió a la muerte de Fernando VII, y que patrocinó el primer gobierno liberal del reinado isabelino; se trataba, en definitiva, de crear cuerpos de técnicos capaces de impulsar el desarrollo industrial que España necesitaba a todas luces.

Para ingresar en la Escuela de Caminos, Canales y Puertos (que entonces dependía de Instrucción Pública, en el Ministerio de Fomento) era necesario pasar un duro examen centrado especialmente en las Matemáticas. En el caso de Echegaray el programa de pruebas a realizar estuvo determinado por una Real Orden de 8 de julio de 1847. Eran estas las materias de las que se examinaba:

a.) Aritmética., Algebra, con inclusión de la teoría y resolución de las ecuaciones superiores y teoría de las cantidades exponenciales y logarítmicas. 
b.) Geometría.- Trigonometría rectilínea y esférica, con el uso de las tablas de logaritmos y líneas trigonométricas para la resolución de los triángulos de una y otra especie.

c.) Aplicación del álgebra a la geometría, incluyendo la teoría de las curvas y superficies de segundo grado, y las curvas de doble curvatura.

d.) Elementos de topografía.

e.) Traducción del francés.

f.) Principios de dibujo.

Con referencia a los libros de texto que estudió Echegaray durante su carrera, tenemos que estos fueron casi exclusivamente franceses; sólo "por casualidad", señaló Echegaray en sus Recuerdos autobiográficos, "estudiábamos alguna Memoria en inglés, o alguna del alemán traducido al francés, y esto en los últimos años... El francés, y siempre el francés, y autores franceses dominaban en la Escuela de Caminos" En cuanto a algunos nombres: "la Geometría de Vincent, el Algebra de Bourdon, la Analítica de Biot, la Geometría analítica de tres dimensiones de Leroy: estos en la preparación. Y luego, dentro de la Escuela, siempre obras francesas, no las traducidas, sino las originales; por ejemplo: los Cálculos de Navier y Duhamel, la Mecánica de Poisson, la Descriptiva de Leroy, el Corte de piedras de Adhémar, la Mecánica aplicada de Poncelet, la Conducción de aguas de Dupuit".

Si nos atenemos a la parte matemática de esta educación, hay que señalar que muy probablemente servía los intereses de una enseñaanza que pretendía formar ingenieros y no matemáticos que contribuyesen a hacer avanzar a la matemática; en otras palabras: los textos matemáticos franceses utilizados en la Escuela de Caminos no eran, en general y especialmente en los primeros tiempos, realmente obras modernas, propias del siglo XIX; hecho este que ya señaló Julio Rey Pastor en su discurso inaugural en la sección 1 a . (Ciencias Matemáticas) del Congreso de Valladolid de la Asociación Española para el Progreso de las Ciencias, cuando manifestaba, revisando la situación de la matemática en España a mediados del siglo pasado:

"Comienza por entonces la importación de obras francesas: los libros de Ciroddle, el Algebra de Lefebure, de Fourcy, la de Bourdon, la Geometría de Vincent, el Cálculo de Navier, el de Cournot..., obras anodinas todas, incapaces de inspirar amor a esta Ciencia en un país que nace á ella. Si alguna obra original existe entre los libros importados, como son los Elementos de Legendre, es del siglo XVIII; y todas, sin excepción, entran de lleno en esa centuria, si nos atenemos a su contenido, aunque lleven fecha posterior. Estas eran las fuentes en que bebían nuestros antepasados, cuando Gauss, Abel y Cauchy habían renovado todo el Análisis; y habían nacido las Geometrías no euclidianas; y la Geometría proyectiva había llegado con Staudt a su completa madurez; y Riemann había creado la moderna teoría de funciones; en una palabra, cuando ya había nacido, no solamente toda la Matemática que conocemo actualmente, sino muchas otras teorías".

El gran mérito de Echegaray sería el que contribuyó, más que ningún otro matemático de los años que van desde, aproximadamente, 1860 hasta 1890, a introducir en España algunas de las nuevas teorías a las que se refería Rey Pastor. Echegaray finalizó sus estudios de Caminos en septiembre de 1853, siendo el número 1 de su promoción y habiendo obtenido la calificación de sobresaliente en todas las asignaturas que cursó. Como recién graduado el título que le correspondió, en el jerarquizado cuerpo de ingenieros de Caminos, Canales y Puertos, fue el de Ingeniero segundo y su primer destino el distrito de Granada. En 1854 comenzó a dar clase en la Escuela de Ingenieros de Caminos haciéndose cargo de la secretaría de la misma. Allí dio clases de matemáticas, estereotomía, hidráulica, geometría descriptiva, cálculo diferencial y física desde ese año hasta 1868. 
"Las Matemáticas fueron, y son [Echegaray escribió estas líneas hacia 1913-1915], una de las grandes preocupaciones de mi vida; y si yo hubiera sido rico o lo fuera hoy, si no tuviera que ganar el pan de cada día con el trabajo diario, probablemente me hubiera marchado a una casa de campo muy alegre y muy confortable, y me hubiera dedicado exclusivamente al cultivo de las Ciencias Matemáticas. Ni más dramas, ni más argumentos terribles, ni más adulterios, ni más suicidios, ni más duelos, ni más pasiones desencadenadas, ni, sobre todo, más críticos; otras incógnitas y otras ecuaciones me hubieran preocupado. Pero el cultivo de las Altas Matemáticas no da lo bastante para vivir. El drama más desdichado, el crimen teatral más modesto, proporciona mucho más dinero que el más alto problema de cálculo integral; y la obligación es antes que la devoción, y la realidad se impone, y hay que dejar las Matemáticas para ir rellenando con ellas los huecos de descanso que el trabajo productivo deja de tiempo en tiempo. Jamás, ni en las épocas más agitadas de mi vida, he abandonado la ciencia de mi predilección; pero nunca me he dedicado a ella como quisiera".

En sus Recuerdos escribió : "Mi cariño y mi simpatía por la nación francesa eran y son naturales, además de ser justos"; y, como ejemplos, citaba los siguientes: "Cauchy, el gran Cauchy, uno de los más admirables genios creadores de las ciencias matemáticas, el de las funciones imaginarias y de la teoría de la luz, después de Fresnel; Legendre, uno de los primeros fundadores de las funciones elípticas, y el autor de la Teoría de los números, obra verdaderamente clásica; Poncelet, el de las propiedades proyectivas; Poisson, el eminente analista; y Chaesles, el eminente geómetra del siglo; y Liouville, autor de tantos trabajos fundamentales y del teorema sobre los números trascendentales; y tantos y tantos autores más... En mis estudios y mis consultas no salía de los Anales de Terquem, del Journal de Liouville, del Journal de la Escuela Politécnica, de los Anales de la Escuela Normal y del periódico oficial: Comptes rendus de la Academia... En fin... en aquella época, para mí el mundo se reducía a dos naciones: España, mi patria; Francia, la patria adoptiva de mi inteligencia y de mis gustos estéticos". Echegaray se casó (el 16 de noviembre de 1857) con la asturiana Ana Perfecta Estrada. Las nuevas obligaciones, a las que se sumó pronto una hija (y algún tiempo después un hijo), le llevaron a intentar conseguir ingresos suplementarios estableciendo una academia particular de matemáticas para preparar a los estudiantes de la Escuela, o a los que querían ingresar en ella. El éxito inicial que obtuvo se vio truncado por una disposición ministerial que declaraba incompatibles la enseñanza privada y la pública. Intentó entonces salir transitoriamente del Cuerpo, abandonando toda posición oficial, pero el Director de la Escuela, y después también el Director de Obras Públicas le negaron el permiso, y él no se atrevió a dejar definitivamente el Cuerpo, solución a la que, por supuesto, podría haberse acogido.

José Echegaray mantuvo una gran actividad hasta su muerte, ocurrida el 14 de septiembre de 1916 en Madrid. Su extensa obra no dejó de crecer en la vejez: en la etapa final de su vida escribió 25 o 30 tomos de Física matemática. Con 83 años comentaba: No puedo morirme, porque si he de escribir mi Enciclopedia elemental de Física Matemática, necesito por lo menos 25 años.

También fue ministro de Fomento y de Hacienda en varios gobiernos (antes, durante y después de la I República) y actor principal en la creación del Banco de España como banco emisor. Formó parte de la comisión que se desplazó a Cartagena para recibir al rey Amadeo de Saboya. Fue también director de la Real Academia de Ciencias y de la Real Academia de la Lengua y primer presidente de la Real Sociedad Matemática Española.

\subsection{Discurso ante la Real Academia de Ciencias Exactas, Físicas y Naturales con motivo de su ingreso el 3 de abril de 1865}


Este discurso es una grito, una queja del abandono en que se encontraba en ese momento la matemática pura en España, posiblemente no sea absolutamente congruente y pueda ser objeto de muchas críticas de diverso tipo, pero estremeció y resonó con una fuerza innegable.

Me permitiré transcribir algunos párrafos : "Historia de las matemáticas puras en nuestra España. Señores: La honra que de esta muy respetable Academia he recibido, honra tan superior a mis méritos, sí méritos hay en mí, que no como justa recompensa sino como bondadoso estímulo debo considerarla, me impone grandes deberes. No abrigo la esperanza de cumplirlos cual corresponde al nombre ilustre de esta corporación, al saber de sus individuos y al brillo de la ciencia moderna de nuestra patria, porque fueran en mí tales esperanzas locas ambiciones; mas si con buen deseo, con celo infatigable pueden suplirse en algo dotes más elevadas, con uno y otro, que es cuanto de mi voluntad depende, procuraré mostrar mi profundo agradecimiento por este elevado título que sin merecer recibo, y que jamás esperé. De estos sagrados deberes hoy cumplo uno, y sólo por cumplirlo voy a ocupar la atención de la Academia: no la merece mi persona; pero la importancia del punto que he escogido, los arduos problemas que encierra y su inmensa trascendencia para el porvenir, le hacen digno de estudio y meditación: que al fin es la ciencia, por más abstracta que en sus concepciones a primera vista parezca, germen fecundo de progreso para pueblos, enérgico purificador del alma, luz que alumbra a la humana inteligencia con divinos resplandores. Voy a ocuparme de la historia de las Matemáticas puras en nuestra España; y entiendo por Matemáticas puras la ciencia eminentemente racional, no la Física ni la Astronomía, ni todas aquellas que, si bien acuden al análisis algebraico o geométrico como a poderoso auxiliar, son por su naturaleza, y por el carácter de los fenómenos que estudian, verdaderas ciencias de observación."

"Ya a fines del siglo VIII, exterminados los omeyas, y triunfantes los abasidas, termina el período de la conquista, y comienzan saborearse las dulzuras de la paz; el gusto por las ciencias se desarrolla entre los árabes; sus feroces instintos se suavizan; el arte y la poesía les eleva a nueva vida; y mil ilustres ingenios hacen de Bagdad la Atenas de Oriente. Mohamed Ben Musa, o hijo de Moisés, que Leonardo de Pisa y el célebre Tartaglia suponen ser el inventor del Algebra, hipótesis falsa, pero que prueba la elevada opinión que del geómetra árabe tenían: Thébit Ben Corrah, fecundo y eminente matemático; Hassen, uno de los tres hijos de Musa, de quien se dice que resolvió problemas geométricos que hubieran sido el asombro de la antigüedad; Alkindi, a quien coloca Cardano, siquiera sea hiperbólico el elogio, entre los doce más claros ingenios que hasta entonces hubiesen ilustrado al mundo, y tantos otros como la historia de aquellos tiempos cita, y cuyas obras, si de los autos de fe se salvaron, en el Escorial o en Toledo o en alguna otra biblioteca descansarán hoy, dan nombre y nombre glorioso a la raza oriental de que proceden.

España fue entonces, pero no la España cristiana, el centro del saber en Europa: en las célebres escuelas de Córdoba, de Sevilla, de Murcia y de Toledo se enseñaba toda la ciencia acumulada durante tantos y tantos siglos en Oriente."

"El siglo XIV fue casi estéril para los descubrimientos matemáticos; como si el mundo antiguo hubiera agotado toda su fuerza creadora en los Euclides, en los Arquímedes y en los grandes geómetras árabes, y cayese al fin rendido y extenuado; o como si la naturaleza, recogida en sí misma, condensase todas sus energías en misterioso y sublime trabajo, preparándose para aquel aborto gigante que dio al mundo un Newton, un Descartes, un Leibniz, un Euler, un Lagrange y todos los admirables analistas de los 
siglos XVI, XVII y XVIII, reinan en Europa por largos años profunda calma, prolongado y solemne silencio."

"La razón humana, en su desarrollo histórico, procede siempre elevándose de lo particular a lo general, de las relaciones sencillas a los grandes principios; y cuando de esta suerte se halla en posesión de una ley, es dueña absoluta de toda la esfera racional que esa ley domina, y de todo lo que en ella corno en su unidad superior se halla encerrado. La sustitución de las letras, símbolos generales, a los números, es la sustitución de la gran categoría de la cantidad a tales o cuales cantidades particulares; y toda verdad que de este modo se demuestre, abarcará cuanto aquella categoría abarca. Por otra parte, si el raciocinio directo sólo puede desarrollarse en serie lineal de silogismos, el Algebra, al contrario, condensa en una expresión analítica todo un razonamiento, y al combinar muchas ecuaciones entre sí o al someterlas a determinadas transformaciones, no opera sobre sencillos silogismos, sino más bien sobre series extensísimas, que une y enlaza, por decirlo así, en múltiples dimensiones."

"¡Qué siglo tan admirable este del álgebra de Viete, la geometría de Descartes y el cálculo de Newton y Leibniz! Gran siglo, sí, para Europa el siglo XVII; mas ¿qué ha sido para nuestra España ¿Qué descubrimiento analítico, que verdad geométrico, que nueva teoría lleva nombre español ¿Quiénes los rivales de Viete, de Fermat, de Pascal, de Descartes, de Harriot, de Barrow, de Brouncker, de Wallis, de Newton, de Huygens, de Gregorio de San Vicente, de Leibniz, de los Bernoulli Yo los busco con ansia en los anales de la ciencia, y no los encuentro; paso impaciente de una a otra historia por si hallo al fin, en alguna, reparación al desdeñoso e irritante olvido en que todas nos dejan; y en todas ellas, bien se echa de ver la nacionalidad del escritor por las cariñosas predilecciones que a sus compatriotas muestra, aparecen los nombres de Francia, Italia, Inglaterra, Alemania, Bélgica, Flandes y Holanda, y en todas se paga tributo de respeto y admiración a los grandes geómetras; pero enninguna encuentro a nuestra España."

"Mancha, y mancha vergonzosa, porque no basta que un pueblo tenga poetas,pintores, teólogos y guerreros; sin filósofos y sin geómetras, sin hombres que se dirijan a la razón, y la eduquen y la fortifiquen y la eleven, la razón al fin se debilita, la imaginación prepondera y se desborda, hasta el sentimiento religioso se estanca y se corrompe: y si por un vigoroso esfuerzo, pueblo que a tal punto llegue no restablece el armónico equilibrio que entre las facultades del alma humana debe siempre existir,morirá, como mueren los pueblos que se corrompen y se degradan, y hasta aquello mismo que fue en otro tiempo su gloria, será en sus postreros instantes su vergüenza y su tormento." "Todos estos pueblos, entre guerras y sangre, y terribles sacudimientos, conservan entera y vigorosa su razón, y de entre el caos y las ruinas se alzan genios potentes, nobles inteligencias, profundos filósofos y grandes geómetras; y en nuestra España, invencible y poderosa, dueña del mundo nuevo, y aspirando a dominar el antiguo, tranquila,relativamente al resto de Europa, en el interior, temida fuera, con su unidad política y su unidad religiosa, sólo se conservan puros, y no siempre, la imaginación y el sentimiento; pero la razón, la facultad más noble del ser que piensa, languidece y decae, y con ella todo languidece y muere al fin."

"Y no he tenido que referir la historia de las matemáticas, allá, para probar que no la hay aquí, y para probarlo, señores, con la elocuente voz de los hechos, demostración ruda pero firmísima, contra la cual se estrellan impotentes, sofismas, alharacas y declaraciones, he necesitado buscar la filiación de cada verdad, el origen de cada teoría, el nacimiento de cada idea, el autor de cada descubrimiento, y después los hombres que desarrollan y perfeccionan aquellos descubrimientos y teorías, formando de esta suerte la ciencia moderna en toda su magnífica riqueza; y he necesitado todo esto para poder 
decir sin remordimiento y sin temor: la ciencia matemática nada nos debe: no es nuestra; no hay en ella nombre alguno que labios castellanos puedan pronunciar sin esfuerzo.

La resolución de las ecuaciones de tercero y cuarto grado se debe a Ferri, a Tartaglia,a Cardan y Ferrari, italianos.

El álgebra, a Viete, francés.

La teoría de ecuaciones, al mismo Viete, francés, y a Harriot, inglés.

La geometría analítica, a Descartes, francés también.

El cálculo diferencial, a Newton y Leibniz, inglés el primero, alemán el segundo.

La teoría de los números y el análisis indeterminado, a Fermat y Bachet de Meziriac, franceses ambos.

Las fracciones continuas, a Brouncker, inglés.

Los logaritmos, a Neper, inglés también.

La geometría superior, a Desargues, francés.

Las series, a Wallis y Mercator, inglés el primero, alemán el segundo.

El cálculo integral a Leibniz, Newton y los Bernoullí. Al francés Monge, la geometría descriptiva. El cálculo de variaciones, al piamontés Lagrange. Y después amplían, desarrollan y perfeccionan estas teorías, o crean otras nuevas, numerosos e insignes geómetras, todos extranjeros a nuestra patria."

"Y ahora, señores, descrita ya a grandes rasgos la historia de las Matemáticas puras, y puesta de relieve por la comparación con otros países, nuestra deplorable decadencia desde el Renacimiento, que fue para España más bien morir que renacer, hasta principios de este siglo, debiera dar por terminada mi tarea; pero hay un punto de suma importancia, íntimamente enlazado con el tema de mi discurso, y sobre el cual he de presentar, siquiera de pasada, algunas reflexiones que recomiendo a la meditación de vuestro elevado pensamiento."

\subsection{EL premio Nobel de Literatura (1904)}

Discurso del representante de la Academia Sueca al referirse a Echegary "II Después del esplendor del teatro griego, es principalmente entre los ingleses y los españoles que se ha desarrollado un arte dramático nacional. Para entender el drama español moderno, es necesario saber qué condiciones en la vida de períodos pasados se encuentran detrás de él. Durante mucho tiempo el drama español ha mostrado fuertes contrastes. Por un lado, hay la floración más lujosa de la fantasía; Por otra, una casuística extremadamente sutil ya veces convencional. En un lugar, hay coloración brillante, y en otro, un gran afecto por la antítesis retórica. El lenguaje enfático se combina con la intriga enredada. Efectos llamativos son violentos, el orden lírico intenso. Las desarmonías son agudas, y los conflictos casi siempre tienen una resolución trágica. La dialéctica es vigorosa. Sin embargo, la vida interior es muy rica, y la severa, Dictados inflexiblemente aplicados de honor no excluyen el lujo de expresiones repentinas de fantasía. En el drama español el artificial ha logrado fundirse con una auténtica originalidad.

El heredero y continuador de estas gloriosas y características tradiciones es el escritor que ha recibido la mitad del Premio Nobel este año. Hijo de la edad moderna y perfectamente independiente en sus juicios, no tiene la misma concepción del mundo que tenía Calderón. Amando la libertad y habiendo luchado a menudo por la tolerancia, no es amigo del despotismo o de la jerarquía, pero todavía hay en él el mismo ardor exótico y la misma dignidad que desde tiempos más antiguos han sido las marcas distintivas de los dramaturgos españoles. Este escritor es José de Echegaray. Al igual que 
sus antepasados, sabe cómo presentar el conflicto, es extremadamente emotivo y muy interesado en diferentes temperamentos e ideales, y como ellos disfruta estudiando los casos más complicados de conciencia. Es maestro completo del arte de producir en la audiencia piedad y miedo, los conocidos efectos fundamentales de la tragedia. Así como en los maestros del viejo teatro español, hay en él una unión sorprendente de la imaginación más viva y del sentido artístico más refinado. Para esto se puede decir de él como un crítico de otra manera antipático a él declaró "que es de pura raza española". Sin embargo, su concepción del mundo es enorme. Su sentido del deber se ha purificado, sus concepciones fundamentales son benevolentes, y su heroísmo moral, aunque conserva un carácter nacional peculiar, tiene las características de una humanidad universal. Hay en él una unión sorprendente de la imaginación más viva y del sentido artístico más refinado. Para esto se puede decir de él como un crítico de otra manera antipático a él declaró "que es de pura raza española". Sin embargo, su concepción del mundo es enorme. Su sentido del deber se ha purificado, sus concepciones fundamentales son benevolentes, y su heroísmo moral, aunque conserva un carácter nacional peculiar, tiene las características de una humanidad universal. Hay en él una unión sorprendente de la imaginación más viva y del sentido artístico más refinado. Para esto se puede decir de él como un crítico de otra manera antipático a él declaró "que es de pura raza española". Sin embargo, su concepción del mundo es enorme. Su sentido del deber se ha purificado, sus concepciones fundamentales son benevolentes, y su heroísmo moral, aunque conserva un carácter nacional peculiar, tiene las características de una humanidad universal.

José de Echegaray nació en Madrid en 1833 pero pasó sus años de infancia en Murcia, donde su padre ocupó la cátedra de Estudios Griegos en el Instituto. Recibió su licenciatura a los catorce años, pronto entró a la Escuela de Ingeniería Civil, donde se distinguió por su aplicación celosa y su habilidad penetrante. Cinco años más tarde, en 1853, él terminó su carrera de ingeniería después de haber compilado un expediente más brillante. La matemática y la mecánica habían sido sus estudios favoritos, y su comprensión singular de estas ramas del aprendizaje le permitió, después de un año, ser nombrado profesor en la misma escuela que había asistido recientemente como estudiante. Parece que durante algunos años su lucha por la existencia fue bastante dura, Y tuvo que dar clases particulares para sostener el modo de vida más modesto. A pesar de todo, pronto se convirtió en un eminente profesor, distinguiéndose a sí mismo en la matemática pura y aplicada, y se convirtió en un ingeniero excepcional. Al mismo tiempo, estudió energéticamente la economía política, abrazando las ideas del libre comercio. Pronto, ese gran talento, ese ingeniero vivaz, fue llamado a las tareas más altas y más grandes. Tres veces ha sido ministro del gobierno de su país. Según los que lo conocen, sean adversarios o amigos, siempre ha mostrado una singular destreza en la administración de las finanzas públicas y de las obras públicas. Y se convirtió en un ingeniero sobresaliente. Al mismo tiempo, estudió energéticamente la economía política, abrazando las ideas del libre comercio. Pronto, ese gran talento, ese ingeniero vivaz, fue llamado a las tareas más altas y más grandes. Tres veces ha sido ministro del gobierno de su país. Según los que lo conocen, sean adversarios o amigos, siempre ha mostrado una singular destreza en la administración de las finanzas públicas y de las obras públicas. Y se convirtió en un ingeniero sobresaliente. Al mismo tiempo, estudió energéticamente la economía política, abrazando las ideas del libre comercio. Pronto, ese gran talento, ese ingeniero vivaz, fue llamado a las tareas más altas y más grandes. Tres veces ha sido ministro del gobierno de su país. Según los que lo conocen, sean adversarios o amigos, siempre ha mostrado una singular destreza en la administración de las finanzas públicas y de las obras públicas.

Podemos comprender fácilmente el asombro general cuando este erudito, que había publicado tratados sobre geometría analítica, física y electricidad, dedicó su infatigable energía a escribir para el teatro. Se ha dicho que sus creaciones para el escenario tenían la forma de ecuaciones y problemas. Si la nueva 
manifestación de su genio fue aclamada con entusiasmo por numerosos admiradores, también encontró críticas severas. Sin embargo, nadie podía negar que sus obras se distinguían por un profundo sentido moral. En cierto modo, los críticos no se equivocaron al sostener que en sus dramas, siguiendo el ejemplo de algunos cirujanos, rara vez utilizaba otro método que el de "urere et secare»; Sin embargo, hay algo que admirar en esta Musa de exaltación romántica y severidad austera que condena cualquier compromiso con el deber.

Despreciando la aprobación transitoria de la moda y escuchando sólo las inspiraciones de su genio, Echegaray persiguió su carrera triunfal, demostrando una fecundidad dramática que nos hace pensar en Lope de Vega y Calderón.

Incluso en su juventud, cuando estaba asistiendo a la Escuela de Ingeniería Civil, se entusiasmó con el drama y utilizó sus ahorros para obtener entradas para el teatro. En 1865 escribió una obra titulada La hija natural , a la que siguió El libro talón de 1874. La obra de teatro llevaba un seudónimo en lugar del nombre del autor, pero no tomó mucho tiempo al público Para adivinar que el aclamado dramaturgo era Echegaray, entonces Ministro de Hacienda de España. Algunos meses despuésLa última noche fue puesta en escena, y desde entonces su imaginación fértil no ha dejado de engendrar creaciones siempre nuevas. Trabaja con tal rapidez que en un año ha publicado tres o cuatro obras. Puesto que la falta de tiempo prohíbe una revisión completa de todas sus producciones, basta con mencionar brevemente algunas que han merecido una atención general. Echegaray anotó su primer triunfo en noviembre de 1874 con el drama La esposa del vengador[Esposa del Vengador], en la que se reveló su verdadero genio y en el que, junto a ciertas exageraciones, se pueden admirar las más grandes bellezas.

El público podía imaginar que había sido llevado de vuelta a la Edad de Oro del drama español, y saludó a Echegaray como el regenerador de la época más brillante de la poesía dramática de la nación. En el puño de la espada, presentado al año siguiente, fue recibido con el mismo aplauso. El poder sublime que se manifiesta en esta noble concepción movió tanto a muchos espectadores que el aplauso no se detuvo con la actuación, y después del último acto Echegaray tuvo que aparecer en el escenario siete veces para recibir la aclamación de la audiencia. Pero grandes controversias surgieron en 1878 cuando,[La Estaca y la Cruz], el poeta se mostró el defensor del libre pensamiento contra la intolerancia, de la humanidad contra el fanatismo. Típico de Echegaray, como él mismo ha observado, es su Conflicto entre dos deberes, que fue presentado en 1882. Un conflicto de deberes se encuentra en casi todos sus dramas, pero rara vez se ha empujado el conflicto a tal Un extremo como en esta pieza. Otros dos dramas han hecho famoso su nombre. Estos dos, excelentes obras inspiradas son O locura ó santidad [ loco o Santo ] y El gran Galeoto [Gran Galeoto], el primero presentado en enero de 1877, y la segunda en marzo de 1881. En O locura ó santidad Hay una gran riqueza de ideas y genio profundo. Muestra a un hombre que, movido por su justicia para sacrificar su prosperidad y bienes mundanos, es considerado loco y tratado como tal por sus amigos y por el mundo en general.

Lorenzo de Avendano renuncia a un nombre ya una fortuna cuando descubre inesperadamente pero innegablemente que no le pertenecen legalmente y persiste en su resolución cuando la única prueba indiscutible de su ilegitimidad ha desaparecido. Tal idealismo es juzgado locura por su familia, y Lorenzo es mirado por todos como un Don Quijote, obstinado y simple-minded. La estructura del drama es firme y sólida, demostrando que es el trabajo de un ingeniero que calcula precisamente todos los elementos que han entrado en él, Pero nos muestra a un grado todavía mayor el poeta del genio creativo maduro. Más que una colisión externa, trata el conflicto interno de una figura extremadamente triste. Consiste en una lucha entre deber y oportunismo, y Lorenzo en seguir el dictado de su conciencia 
alcanza el martirio. La experiencia siempre ha demostrado que con mucha frecuencia el que obedece fielmente a su conciencia debe estar preparado para soportar el destino de un mártir.

El gran Galeoto hizo una impresión aún mayor. En el primer mes después de su apertura, pasó por no menos de cinco ediciones e inspiró una suscripción nacional para honrar a su autor. Debido a la interpretación magistral de la psicología de los personajes, la obra tiene un valor duradero. Muestra el poder de la calumnia. El rasgo más inocente es desfigurado y escandalosamente deformado por los chismes de la gente. Ernesto y Teodora no tienen nada que reprocharse a sí mismos, pero el mundo los cree culpables, y al fin, abandonados por todos, terminan arrojándose los unos a los otros. La sutileza del análisis psicológico se revela con tal detalle magistral de la observación que esos dos espíritus nobles, de ninguna manera deseosos de robar el derecho de su vecino, se enamoran mutuamente sin sospecharlo. Ellos descubren el hecho de su amor sólo por medio de la persecución a la que se ven expuestos. El romanticismo triunfa en este drama cuya belleza poética es claramente perceptible, cuyos detalles líricos poseen un color deslumbrante, y cuya estructura es sin defecto. Echegaray sigue trabajando como dramaturgo. Este año (1904) ha publicado una nueva obra, La desequilibrada , cuyo primer acto es una auténtica obra maestra de exposición e individualización y que en su totalidad no revela debilitamiento de la inspiración poética. En esta obra se nos muestra a Don Mauricio de Vargas, un tipo claro de esa caballería tan querida a Echegaray, esa caballería que no quiere comprar ni siquiera su propia felicidad a costa de un deber comprometedor. De este modo es justo que el Premio Nobel sea otorgado a este gran poeta cuya producción se distingue por su energía viril y cuyo modo de ver está impregnado de ideales tan altos que con una razón abundante ha podido decir de él un eminente crítico alemán: "Er verlangt Recht und Pflichterfüllung unter Allen Umständen."

Echegaray ha puesto en la boca de uno de los personajes de el gran Galeoto las palabras más pesimistas sobre el mundo, que "no reconoce las sutilezas del genio hasta tres siglos después de su muerte». Sin duda esto puede suceder. Pero contra la aplicación general de la tesis anterior podemos ofrecer la admiración justificada que la obra de Echegaray ha despertado. A esos homenajes de agradecimiento la Academia Sueca ha aceptado añadir todavía una más, otorgando el Premio Nobel en homenaje al célebre poeta, honor y gloria de la Academia Española, José de Echegaray".

En el banquete, CD af Wirsén señaló que compartir el Premio no disminuyó de ninguna manera el valor de los laureados. Recordó las obras puras, limpias y frescas de Frédéric Mistral, nombrando las principales y pidiendo al ministro de Francia, el señor Marchand, que transmitiera al famoso poeta provenzal el homenaje que la Academia sueca y todos los reunidos Tomó placer en rendirlo. El orador entonces repasó la obra imponente de Echegaray y expresó sus arrepentimientos por su estado de salud y explicó que el Ministro de España había sido impedido de asistir a este banquete y de recibir las felicitaciones por su famoso paisano.

El Ministro de Francia, Sr. Marchand, respondió al Secretario de la Academia Sueca y recordó que en el año anterior les había agradecido el Premio otorgado al Sr. y Sra Curie; Esta vez habló por el gran poeta de quien Provenza está justamente orgulloso. Habló de un acontecimiento muy conmovedor. Hace cuarenta y cinco años la Academia Francesa, que no tenía a su disposición recursos tan grandes como los que el gran Nobel había dotado a la Academia Sueca, decidió, a sugerencia de Lamartine, entusiasta de Mirèio, otorgar el premio De 3000 francos a Mistral. Cuando le preguntaron al autor, que llevaba una vida sencilla en el campo, qué haría con el Premio, contestó: "Es un premio para la poesía; No es para ser tocado!». El modesto poeta compartía su "superabundancia» con los demás. El Sr. Marchand también actuó como portavoz de su colega, el Ministro de España, para expresar la 
gratitud del Sr. Echegaray."

Echegaray escribió más de 60 obras de teatro, muchas de ellas en verso. La generación del 98 no lo aceptó como un gran escritor, en la actualidad sus obras son muy pocas veces representadas, Valle Inclán lo llamaba el viejo idota e incluso estando grave no aceptó la oferta de recibir sangre de Echegaray pues este hablaba en gerundios. El gran José Martí asistió a una obra de Echegaray y comentó su obra. El 21 de junio de 1879 en el Liceo Artístico y Literario de Guanabacoa José Martí pronunció un discurso acerca de José Echegaray. Esa intervención no se conserva y sólo aparecen en el tomo 15 de sus Obras Completas varios fragmentos, algunos de los cuales son referencias hechas por Martín Pérez en una crónica publicada en La Patria, el 29 de junio del citado año. También en unos trabajos que publicó en 1875 en la Revista Universal, de México comentó en torno a obras de este creador.

Martí comentó sobre la titulada La esposa del vengador de este creador español y expresó que ese drama presentaba un argumento nuevo, grandiosamente concebido, sonoramente rimado, dispuesto con más osadía que práctica y pensado y hablado con gran fuerza de creación y atrevimiento. Añadió seguidamente que tenía defectos graves y también bellezas a raudal. Consideró que los defectos eran de artificio, de disposición y que aún en ellos no se encontraba nada vulgar. Seguidamente se refirió a cómo se desarrollaba la obra formada por tres actos. Explicó que el drama descansaba en la perdida de la vista de la protagonista y el desenlace estaba asociado con la recuperación de su visión.

Martí opinó sobre el manejo del tiempo entre un acto y otro, y al respecto manifestó en la crítica que hiciera a la obra La esposa del vengador de José Echegaray: "Algo hay grave en la obra, y es la licencia de tiempo que en ella se ha tomado el autor. Tan hermoso es el drama, que entusiasma a pesar de este defecto; pero el defecto es grave en sí." Llamó la atención del salto que se había producido entre el final del primer acto y el comienzo del segundo, en que de pronto sin previa explicación el espectador se encuentra que han transcurrido tres años. Y dijo al respecto: "Falta indudablemente en el drama, la unidad de tiempo; no la que rigurosamente exigen retóricos y preceptistas, sino la que quiere racionalmente el instinto de la verdad."

Y añadió más adelante: "Se disculpa y se perdona, pero no se justifica la falta de unidad en este drama. Aquí la concepción grandiosa no alcanzó la realización hábil: de allí el defecto." Calificó a esta obra como extraña, grande, defectuosa y exclusivamente estética y planteó que sus defectos son más notables que su misma originalidad. Detalló más adelante al exponer otras consideraciones tanto positivas como negativas sobre este drama: "Hay violencia en algunas situaciones; pero hay extraordinario atrevimiento en numerosas escenas, en que de súbito y vigorosamente pone en contraste caracteres por completo distintos. Es lírica porque lo pide así su clase de belleza. No es el lirismo de Echegaray la armonía vana de una versificación fácil y abundante; es el lirismo del concepto; es la filosofía en la poesía; es el sentimiento expresado en imágenes bellas."

Finalmente dijo que consideraba buena, bella y grandiosa la obra irregular de Echegaray. Y agregó: "La amo porque me ha hecho gozar, y la estimo porque a un tiempo obraron en ella un gusto exquisito, el hábito del bien hablar, una fuerza poderosa de creación, y unas inconformidades con la existencia que dan alta medida del rebelde espíritu y del potente entendimiento del autor." No fue esta la única ocasión que Martí hiciera referencia y expusiera valoraciones sobre obras de José Echegaray. 


\subsection{El aporte matemático de Echegaray}

Como profesor de la Universidad Central (que después se convertiría en la Complutense) enseño los cursos usuales sobre todo cálculo diferencial e integral. En el Ateneo dictó por varios años una serie de temas sobre física matemática, la obra digitalizada consta de varios miles de páginas. Escribió varios artículos interesantes sobre cuadratura del círculo y el método de Wantzel y como no tenía a mano la de demostración de Lindemann de la trascendencia de $\pi$, la reconstruyó. En el curso en el Ateneo 1896-97 expuso la Teoría de Galois por primera vez en España.

La modernización de las matemáticas en España tiene en Echegray a su Quijote.

\section{Bibliografía}

[1] Echegaray, José. Disertaciones matemáticas sobre la cuadratura del círculo, Madrid,1987. Se puede leer digitalmente en https://rodin.uca.es/xmlui/bitstream/handle/10498/7163/33925574.pdf

[2] Sánchez Ron. (2003) "José Echegaray, matemático." La Gaceta de la RSM. Vol6.3 págs 743-764 . En la red http://gaceta.rsme.es/english/abrir.phpid=62

[3] Sánchez Ron. (2004) "José Echegaray: entre la ciencia, el teatro y la política" Arbor, Vol 179, No 707/708. En la red http://arbor.revistas.csic.es/index.php/arbor/article/view/510

[4] Sánchez Ron. "José Echegaray (1832-1916) el hombre polifacético". Técnica, ciencia, política y teatro en España. Fundación Juanelo Turriano, 2016. Libro escrito con motivo del centenario de la muerta de Echegaray.

[5] Echegaray, José. "Conferencias sobre física matemática Entre 1906 y 1914". http://bdh.bne. es/bnesearch/CompleteSearch.dovisor=\&text=\&field1val=\%22Echegaray\%2c+Jos\%c3\%a9\%22\& showYearltems $=\&$ field $10 p=A N D \&$ numfields $=1 \&$ exact $=0$ on \& text $\mathrm{H}=$ \&advanced=true \&field $1=$ autor \& completeText $=\&$ pageSize $=1 \&$ pageSizeAbrv $=30 \&$ pageNumber $=28$

En el sitio de la Biblioteca Digital Hispánica se encuentran muchas obras de José Echegaray. 